\title{
DEVELOPMENT AND CHARACTERIZATION OF HYBRID COCONUT/GLASS FIBERS REINFORCED LOW DENSITY POLYETHYLENE COMPOSITES FOR BUMPER APPLICATION
}

\author{
Akinlabi, O. David ${ }^{1}$, Ibeh, S. Chukwuemeka ${ }^{1 *}$, Enegide, E. Osther ${ }^{l}$, \\ Garba, N. Salihu ${ }^{2}$ \\ ${ }^{I}$ Technical Services Department, Scientific Equipment Development Institute, \\ Minna-Niger State, Nigeria \\ ${ }^{2}$ Manufacturing Department, Scientific Equipment Development Institute, \\ Minna-Niger State, Nigeria
}

Received 05.11.2019

Accepted 12.03.2020

\begin{abstract}
The EU's End of Life Vehicles (ELV) regulations are forcing car manufacturers to consider the environmental impact of their production and possibly shift from the use of synthetic materials to the use of agro-based materials. However, poor mechanical properties and certain manufacturing limitations currently limit the use of agro-based materials to non-structural and semi-structural automotive components. This research is focused on a composite of hybrid coconut/glass fiber as reinforcement in recycled low density polyethylene matrix alone to enhance the desired mechanical properties for car bumper as automotive structural components. X-ray fluorescence analysis conducted on coconut fiber showed the presence of silica and alumina materials make coconut fibre a choice one. Morphology analysis was performed using scanning electron microscopy (SEM), which reveals that there are small discontinuities and reasonably uniform distribution of the reinforcement fibers and the reinforced low density polyethylene (RLDPE) binder resulting to better mechanical properties. Physic-chemical properties that directly affect developed composite such as variation of Density, Water Absorption, Tensile Strength, Bending strength, Modulus of rupture, Impact Strength and Hardness Values were investigated for both unhybridized and hybridized developed composite. The study shows the successful development of composites of coconut fiber (CF) hybridized with glass fiber (GF) and reinforced low density polyethylene (RLDPE) binder using a simple molding technique. Hybridized samples (CF-GF/RLDPE) showed higher strength when compared to un-hybridized (CF/RLDPE) composites. Better microstructural bonding exists with $25 \%$ and $30 \%$ wt CF-GF composite resulting in good mechanical
\end{abstract}

*Corresponding author: Ibeh Stanley Chukwuemeka, stanleybyhisgrace@yahoo.com 
properties for the hybridized composites. The grades of composites obtained in the course of this study are applicable in the production of low strength car bumpers.

Keywords: composite; coconut fiber; glass fiber; car bumper; low density polyethylene.

\section{Introduction}

In its most basic form, a composite material is one which is composed of at least two elements working together to produce a material whose properties are different from the properties of those elements on their own.

In practice, most composites consist of a bulk material (the matrix) and a reinforcement material, usually in fiber form, which is added primarily to increase the strength and stiffness of the matrix. A variety of materials are being used ranging from lower performance glass fiber/polyester, used in small sailboats and domestic products, to high-performance carbon fiber epoxy systems used in military aircraft and spacecraft. Composite materials offer great potential in reducing vehicle weight, thus increasing fuel efficiency and reducing carbon dioxide $\left(\mathrm{CO}_{2}\right)$ emissions.

In addition to weight reduction, the number of individual parts can be significantly reduced, making the high-volume composite car concept cost-effective [1,2]. Natural fiber composite offers significant opportunities for renewable, biodegradable, and recyclable materials and from sustainable sources at the same time. The transportation industries are utilizing increasing amounts of natural fiber- reinforced plastics in an effort to decrease vehicle weight and boost fuel efficiencies. Despite these advantages, undesirable mechanical properties such as low impact strength and their hydrophilic nature are limiting their application to non-structural and semi-structural automotive components. Hybridization with glass fiber is one prospect to improve the mechanical properties of natural fiber composites [3].

A lot of research has been carried out in the area of composite development. The automotive industry is investigating a host of new applications. Material developers are always faced with the challenges of creating materials with excellent mechanical properties and of low density, thus the need for continuous research in composite material development became imperative. The development of car bumper systems using sheet molding compound (SMC) for automobiles was reported in the literature by Cheon et al., 1995 [2]. Minaudo et al. (1997) developed a one-piece, injection mold thermoplastic rear bumper system with pole impact protection [4]. Clark et al. (1991) described their extensive work on bumper beams using continuous glass fiber composites to study the stress contour in for a passenger car. The material used was glass fiber epoxy composite material, except for the elbow section [5]. I-section beam with $40 \%$ chopped glass fiber GMT (glass mat thermoplastic) was developed and discovered that the bumper design significantly improved the static load and the dynamic impact performance of mineral filed/chopped glass fiber GMT, in the development [6]. Rawson (1999) evaluated the performance of polyolefin in comparison with engineering thermoplastics for blowmolded bumper beams for mid-size vehicles [7]. Suddin et al. (2004) selected polymeric 
based composite materials because of low weight, high specific stiffness, high specific strength, high-energy absorption, and easy to produce in complex shapes to produce bumper fascia for Proton Iswara1.3sAeroback [8]. A conceptual design approach was used in the development of a fiber reinforced epoxy composite bumper system. The researcher described the use of the composite in energy absorption in car bumper as a pedestrian energy absorber, structural composite bumper for medium and heavy-duty truck and bus was developed using a hybrid glass fiber, which is lighter than its steel equivalent [9]. In this research, a hybrid of coconut and glass fibers were utilized as the reinforcement with recycled low density polyethylene (RLDPE) waste using physicomechanical and microstructural property as criteria.

\section{Methodology}

The materials used in this work are reinforced low density polyethylene (RLDPE) as a binder while natural Coconut fibers (CF) and continuous Glass fiber (GF) strands of ASTM D578 / D578M - 18 standard, was used as reinforcements. Figure 1 (a) and (b) shows the extracted coconut fiber before further processing and low density polyethylene after washing thoroughly before shredding.

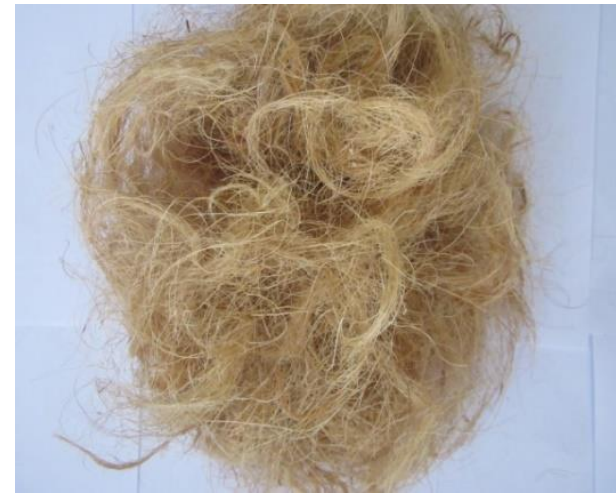

(a)

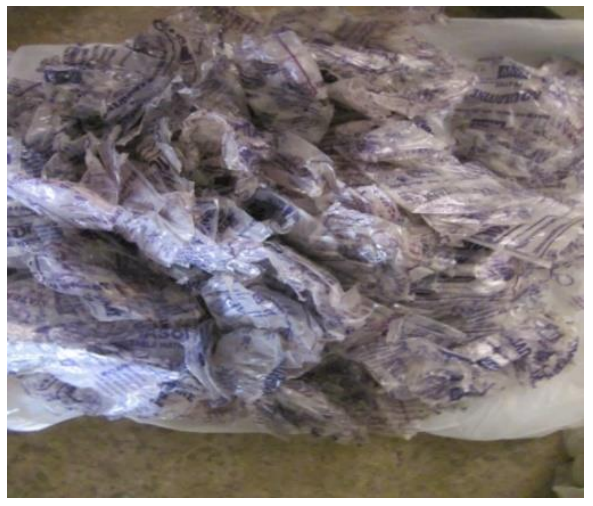

(b)

Fig. 1. (a) Coconut fiber and (b) Low density polyethylene sachet water bags

Waste low density polyethylene sachet water bags were collected from the streets of Minna, Nigeria. They were washed, dried, and cut into small pieces with the aid of a film shredder in the Nigerian Institute of Leather and Science Technology (NILEST), Zaria. The glass fiber was purchased from an engineering store and identified by a metallurgist from the federal university of Technology Minna, Niger state, Nigeria.

\section{Extraction of the Coconut Coir}

The coconut fibers were obtained from a farm settlement center along IlorinIbadan expressway in Ogbomosho, Oyo State Nigeria. The procedure for the coconut coir extraction was based on literature [10]. The mature coconut coir obtained from the farm was cut to a length of $30 \mathrm{~cm}$ each and sliced longitudinally into four pieces and completely submerged in water for 15 days, after which the coir was removed from the water and 
loosened by lapping back and forth in a pool of tap water. They were subsequently sundried for eight hours and further loosened by manual combing. The extracted fibers were then treated with $5 \%$ sodium hydroxide $(\mathrm{NaOH})$ solution for four (4) hours under total immersion condition to avoid oxidation of the fibers and then washed in overflowing tap water until neutral $\mathrm{pH}$ was attained. The treated fibers were dried in an oven for 24 hours at $105{ }^{\circ} \mathrm{C}$ to remove free water and subsequently stored separately in an airtight container. The treated fibers were then used for this study.

\section{Sample Preparation}

Metal Molds were used in the production of the coconut composite samples. Each mold had a cavity that accommodated the composite samples. The dimensions and shapes of cavities were made according to the size and shape of the samples using ASTM Standard D 638-90 for tensile testing and ASTM Standard D 790-97 for flexural testing (ASTM, 1990). The fibers (random oriented) and the binder were mixed by compounding into a homogenous mixture using two roll mills at $130{ }^{\circ} \mathrm{C}$. The compounded mixture was compressed at $150{ }^{\circ} \mathrm{C}$ and a pressure of $10 \mathrm{MPa}$ for 15 minutes to form the bumper composites. Composites were developed with 5, 10, 15, 20, 25, and $30 \%$ (by weight) of fibers. The coconut fiber (CF), glass fiber (GF), and the RLDPE were formulated according to Tables 1-2.

Table 1: Formulation of the composites using CF/RLDPE

\begin{tabular}{lcc}
\hline S/n & Coconut fibers (wt\%) & RLDPE (wt $)$ \\
\hline 1. & 0 & 100 \\
2. & 5 & 95 \\
3. & 10 & 90 \\
4. & 15 & 85 \\
5. & 20 & 80 \\
6 & 25 & 75 \\
7 & 30 & 70 \\
\hline
\end{tabular}

Table 2: Formulation of the hybrid composite using CF-GF/RLDPE

\begin{tabular}{lccc}
\hline S/n & Coconut fiber (wt\%) & Glass fiber & RLDPE(wt\%) \\
\hline 1. & 0 & 0 & 100 \\
2. & 2.5 & 2.5 & 95 \\
3. & 5.0 & 5.0 & 90 \\
4. & 7.5 & 7.5 & 85 \\
5. & 10 & 10 & 80 \\
6 & 12.5 & 12.5 & 75 \\
7 & 15 & 15 & 70 \\
\hline
\end{tabular}




\section{Chemical and Morphological Analysis}

The elemental composition of the coconut fibers was determined using X-ray fluorescence (XRF) analysis in the Multi-User Science Research laboratory, Ahmadu Bello University Zaria, Nigeria. The scanning electron microscope (SEM) JEOLJSM6480LV was used to identify the surface morphology of the composite samples. Samples were washed, cleaned thoroughly, air-dried, and coated with 100 Åthickplatinumin JEOL sputter ion coater and SEM observed at20 kV. Samples were sputter-coated with gold to increase surface conductivity.

\section{Physio-chemical Characteristics}

Test samples were cut from the composites for the mechanical and physical tests according to the recommended Standard for each test. The following tests were carried out:

\section{Density Determination}

A clean sample was weighed accurately in the air using a laboratory balance and then suspended in water. The weight of the sample, when suspended in water was determined, the volume of the sample was determined from the effect of displacement by water (Archimedean principle).

The density of the sample was estimated from the equation below:

$$
\text { Density = Mass } / \text { Volume }
$$

\section{Water Absorption (WA) Test}

This was carried out in order to determine the water absorption of the composite as a result of direct contact and exposure to free water. Specimens with dimensions of $50 \mathrm{~mm}$ x $50 \mathrm{~mm}$ were prepared for evaluation of the water absorption. The test specimens were placed in water in parallel for $30 \mathrm{~min}$ and soaked for 24 hours before further measurement of the weight of the soaked samples. The values of the water absorption as percentages were calculated [11].

$$
W A=(W t-W o) / W o \times 100 \%
$$

Where:

WA $(t)$ is the water absorption (\%) at time t, Wo is the initial weight, and Wt is the weight of the sample at a given immersion time $t$.

\section{Tensile Test}

Tensile strength indicates the ability of the composite material to withstand forces that pull it apart as well as the capability of the material to stretch prior to failure. The ASTM standard test method for tensile properties of polymer composites with the designation D3039-76 was used and to measure the instantaneous applied load and the resulting elongations simultaneously using an extensometer. 
The Tensile strength was calculated from the formula below (ASTM, 1990):

$\sigma=P /(b \times h)$

Where:

$\sigma=$ Tensile Strength, $\mathrm{P}=$ axial load, $\mathrm{b}=$ gauge width, and $\mathrm{h}=$ gauge thickness

\section{Static Bending Test}

The breaking point of the composite sample and its elongation was determined by this test. A static bending test (dry) was conducted using the universal materials testing machine on specimen size $150 \mathrm{~mm}$ x $50 \mathrm{~mm}$ x $4 \mathrm{~mm}$, according to the American Society for Testing and Materials standard D1037. A concentrated bending load was applied at the center with a length 15 times the thickness of the specimen. The bending modulus of rupture (MOR) was calculated according to the following formula:

$$
M O R=3\left(P_{b} \times L\right) / 2\left(b \times h^{2}\right)
$$

Where:

$\mathrm{P}_{b}$ is the maximum load $(\mathrm{N}) ; b$ is the width of the specimen $(\mathrm{mm}) ; h$ is the thickness of the specimen ( $\mathrm{mm})$, and $L$ is the span (mm).

\section{Impact Strength}

The impact test of the composite samples was conducted using a fully instrumented Avery Denison test machine, model number " $\mathrm{C}_{\mathrm{at}} \cdot \mathrm{N}_{\mathrm{r}} \cdot 412$ " of capacity 15 25J. A Charpy impact test was conducted on notched samples. Standard square impact test samples measuring $80 \times 10 \times 10 \mathrm{~mm}$ with a notch depth of $2 \mathrm{~mm}$ and a notch tip radius of $0.02 \mathrm{~mm}$ at an angle of $45^{\circ}$ were used (ASTM, 1990).

\section{Hardness Test}

The hardness of a composite is the relative ability of the material surface to resist indentation by an indenter of a specified dimension under a specified load. Hardness tests of the bumper samples were done to determine the materials' ability to resist plastic deformation. Enerpac Universal Materials Testing machine (BS903 part A 26) (ASTM, 1990) was used using a $1.56 \mathrm{~mm}$ steel ball indenter, with a minor load of $10 \mathrm{~kg}$, and a major load of $100 \mathrm{~kg}$.

\section{Result and Discussion}

\section{Chemical Composition}

The microstructures of the coconut fiber (Figure 2) revealed that the coconut fibers are solid in nature but irregular in size, while the microstructure of the RLDPE matrix of (Figure 3) reveals a chain of lamellae and interlamellar amorphous structure with linear boundaries between adjacent spherulites boundaries. 


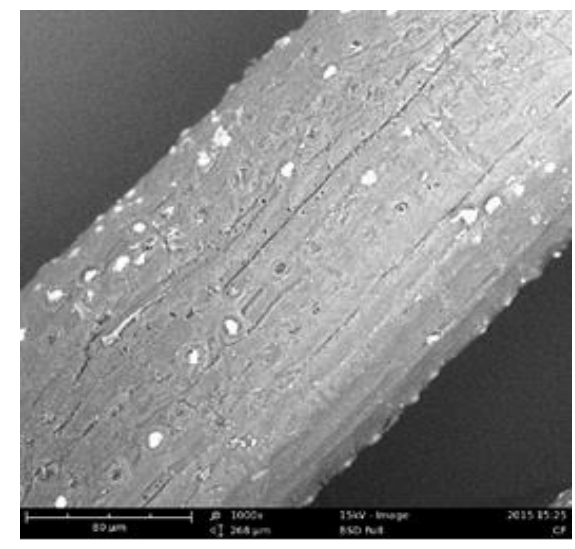

(a)

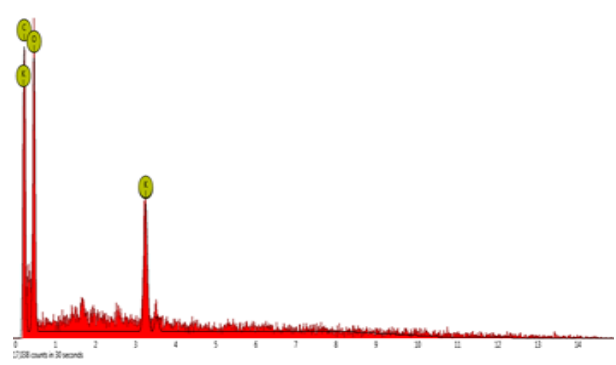

(b)

Fig 2. SEM Microanalysis of coconut fiber (a) Image (b) EDS

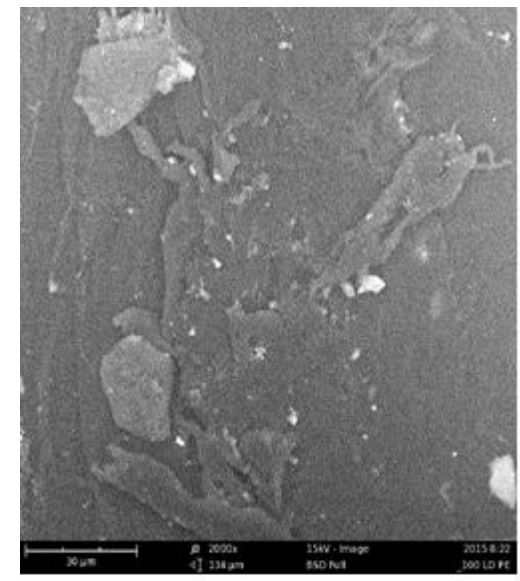

(a)

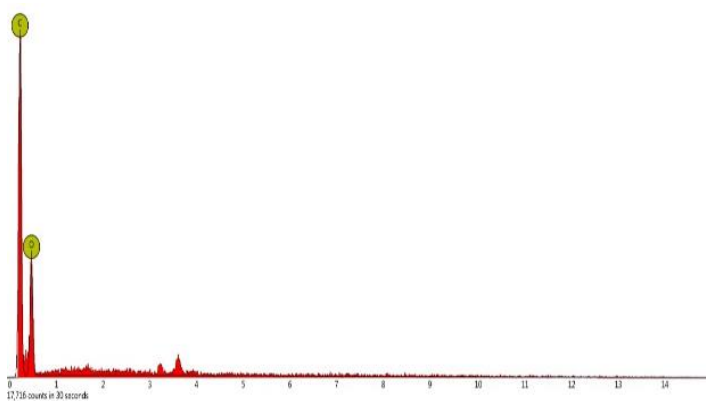

(b)

Fig. 3. SEM Microanalysis of RLDPE (a) Image (b) EDS

Microstructural analysis and comparison of both hybridized (CF-GF/RLDPE) and un-hybridized (CF/RLDPE) fiber-reinforced composites using SEM clearly shows the morphologies of the car bumper composites as shown in Figure 4(a-i). 


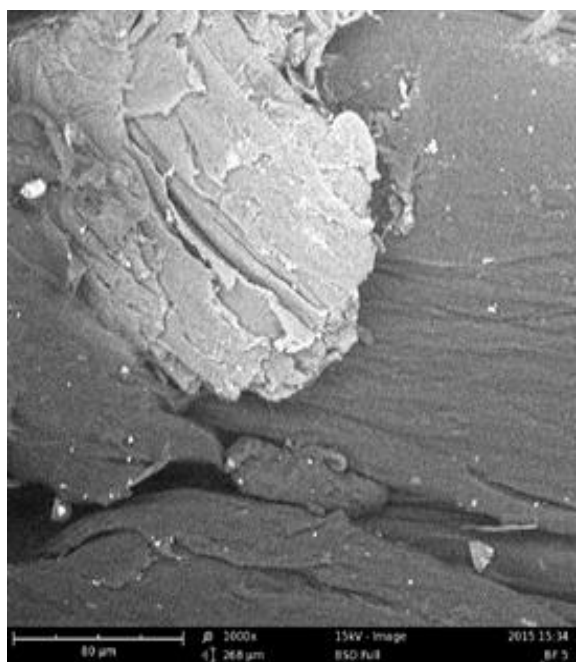

(a)

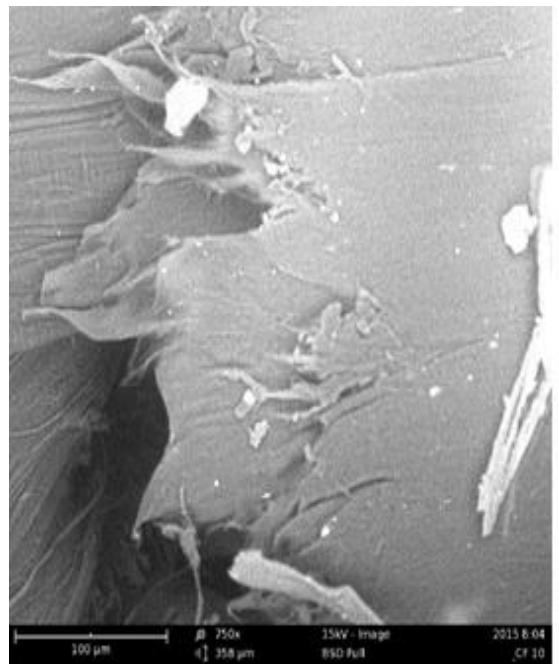

(c)

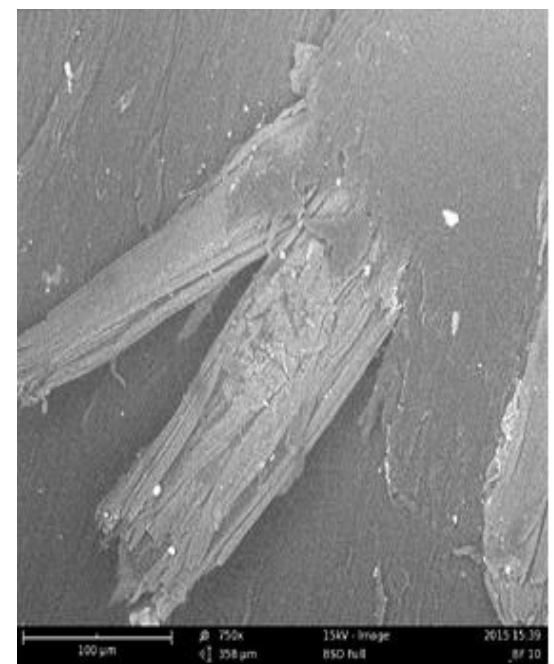

(b)

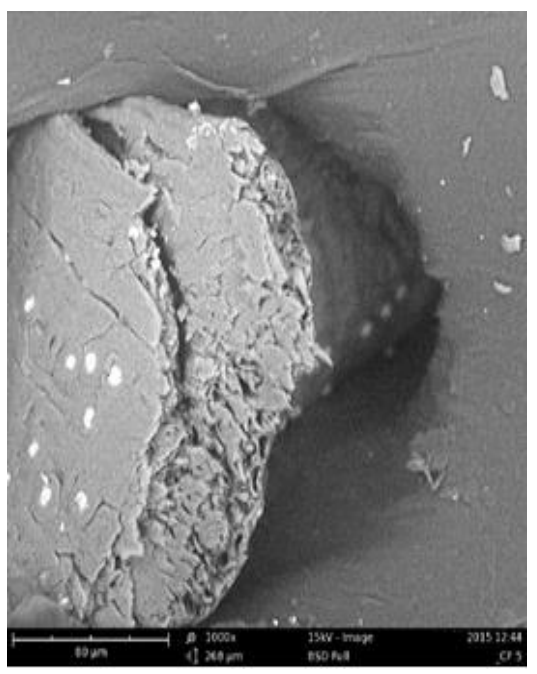

(d) 


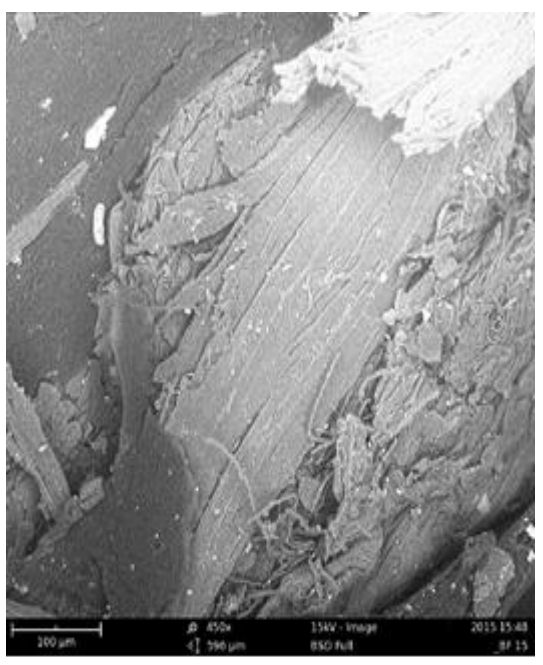

(e)

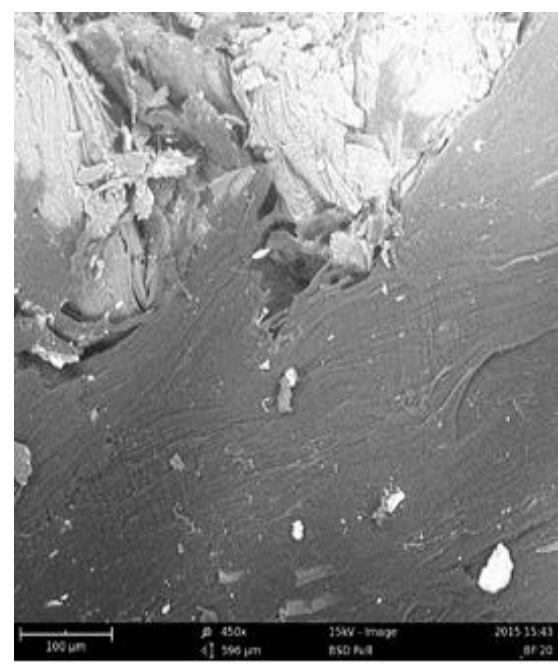

$(g)$

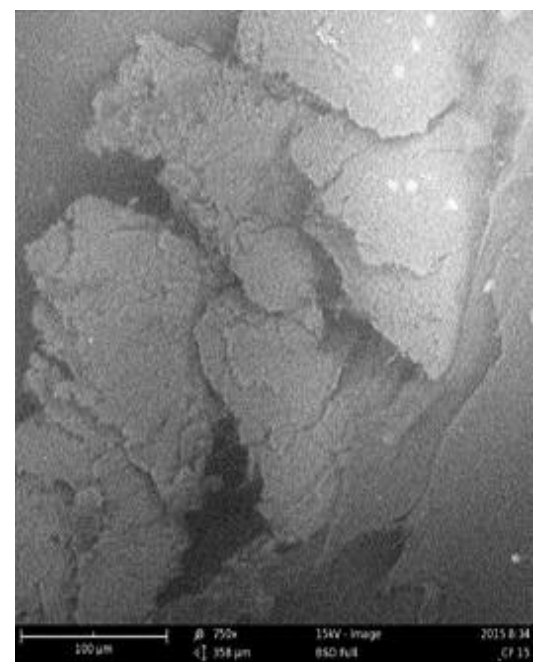

(f)

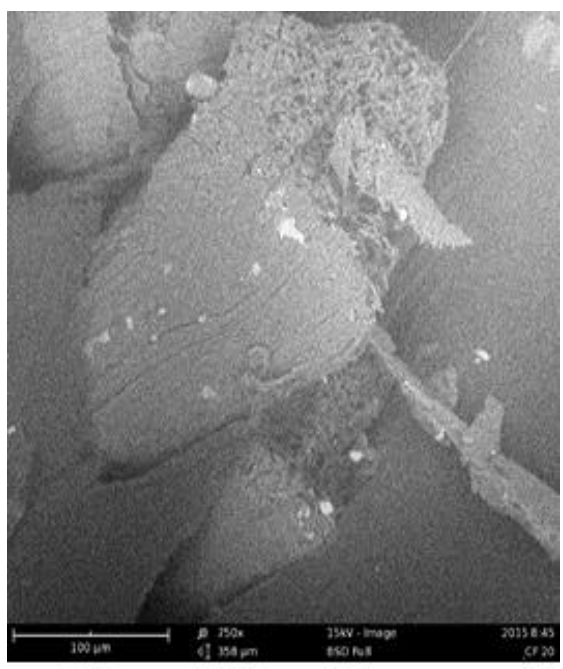

(h) 


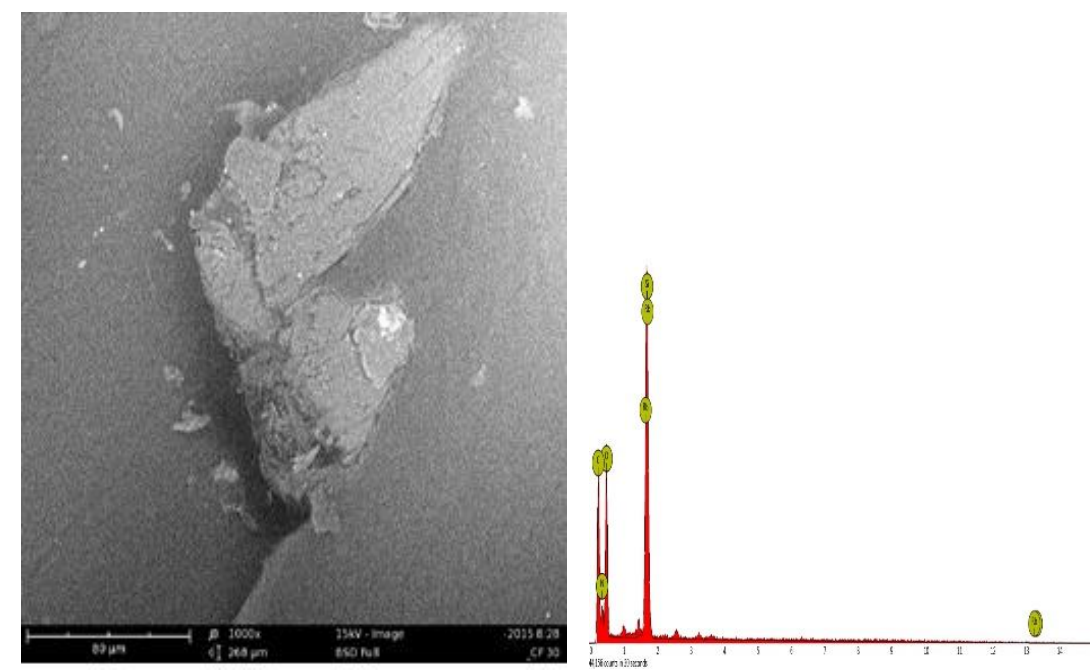

Fig. 4. SEM Microanalysis of (a) $5 \%$ wt. CF composite, (b) 5\% wt. CF-GF hybrid composite, (c) $10 \%$ wt. CF (d) 10\% wt. CF-GF hybrid composite, (e) 15\%wt. CF, (f) $15 \%$ wt. CF-GF hybrid composite, (g) $25 \%$ wt. CF, (h) $25 \%$ wt. CF-GF hybrid composite, (i) $30 \%$ wt. CF-GF hybrid composite

There is a significant difference in the microstructure of hybridized composite compared to un-hybridized composite structures. The microstructure reveals that there are small discontinuities and reasonably uniform distribution of the reinforcement fibers and the RLDPE binder. The reinforcement fibers phase is shown as a white phase, while the binder phase is dark. The fibers are embedded within the amorphous matrix composed of randomly distributed matrix planar boundaries. Fig $4(a, b)$ shows the microstructure of $5 \%$ wt $\mathrm{CF}$ and $5 \% \mathrm{CF}-\mathrm{GF}$ hybrid composite. Unlike the 5\% wt CF composite a large amount of binder phase is observed with 5\% wt CF-GF hybrid composite, which accounts for its better hardness, tensile strength. This was not consistent with $10 \%$ wt CF-GF hybrid composite. Better interfacial bonding between reinforcement and matrix was observed with 15, 25 and 30\% wt CF-GF hybrid composite leading to good mechanical properties for the hybridized composites.

\section{$X$ - Ray Fluorescence Analysis}

The mineral composition using XRF analysis is shown in Table 3, for coconut fibers, and the corresponding spectra for all conditions depicted in Figure 5. Chemical analysis by XRF shows that alumina $\left(\mathrm{Al}_{2} \mathrm{O}_{3}\right)$, silica $\left(\mathrm{SiO}_{2}\right), \mathrm{CaO}, \mathrm{K}_{2} \mathrm{O}$, and $\mathrm{P}_{2} \mathrm{O}_{5}$ were found to be major constituents. Silica and alumina are known to be among the hardest substances. Some other oxides such as $\mathrm{MgO}, \mathrm{Fe}_{2} \mathrm{O}_{3}$, and $\mathrm{TiO}_{2}$ were also found to be present in traces. The presence of hard substances like Silica and Alumina suggests that coconut fibers can be used as particulate reinforcement in RLDPE matrix. 
Table 3: Mineral Composition of Coconut Fibre using XRF

\begin{tabular}{lc}
\hline Element & Concentration $(\mathrm{Wt} \%)$ \\
\hline $\mathrm{Na}_{2} \mathrm{O}$ & 3.065 \\
$\mathrm{MgO}$ & 1.601 \\
$\mathrm{Al}_{2} \mathrm{O}_{3}$ & 20.377 \\
$\mathrm{SiO}_{2}$ & 12.346 \\
$\mathrm{P}_{2} \mathrm{O}_{5}$ & 1.156 \\
$\mathrm{~K}_{2} \mathrm{O}$ & 2.005 \\
$\mathrm{CaO}$ & 1.243 \\
$\mathrm{TiO} 2$ & 1.396 \\
$\mathrm{Fe}_{2} \mathrm{O}_{3}$ & 6.404 \\
\hline
\end{tabular}

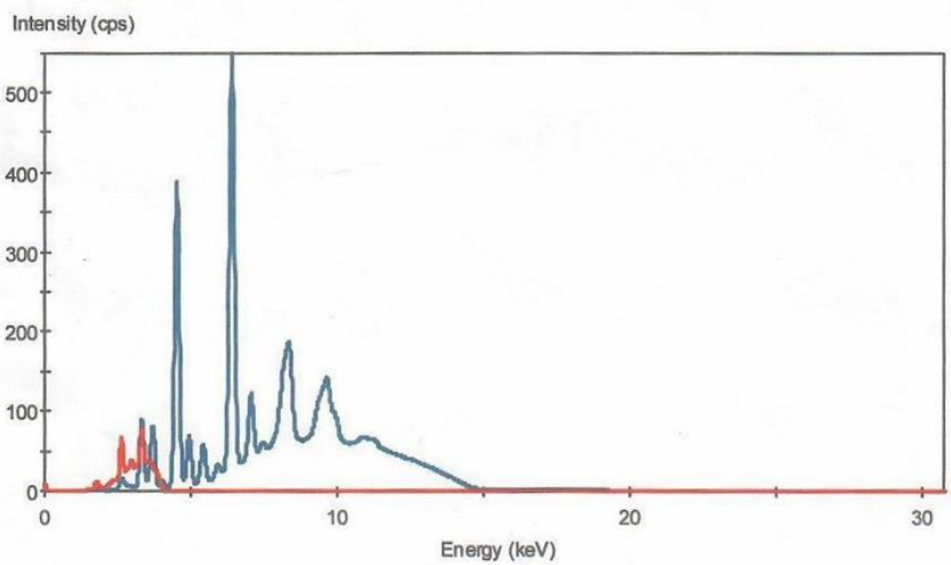

Data series

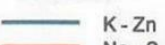

Fig 5. XRF Spectra for CF

Figure 6. illustrates the variation of density concerning the percentage composition of the fibre.

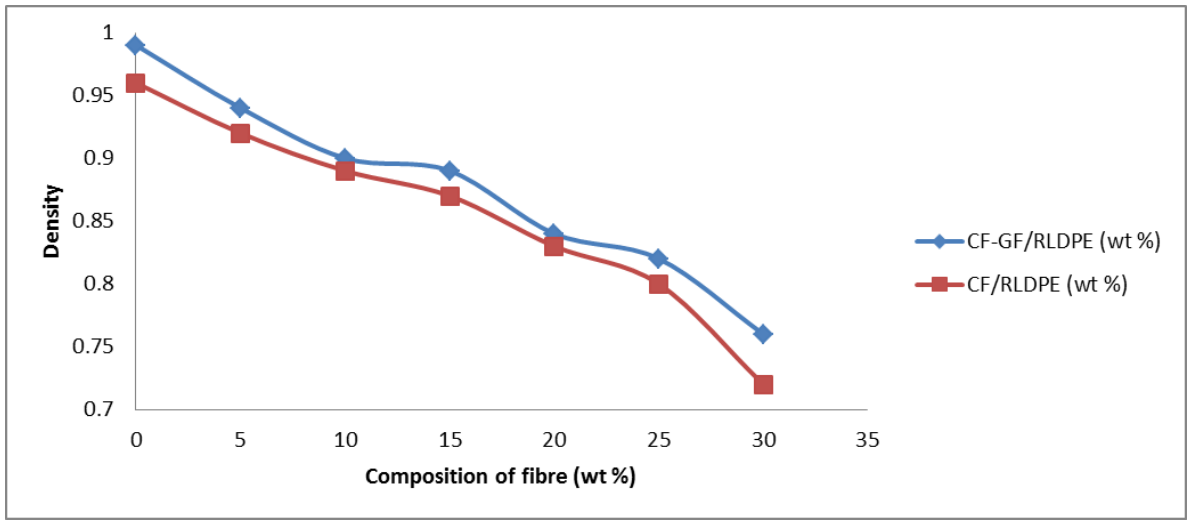

Fig 6. Variation of density versus percentage composition of the fibre 
The results reveal from (Figure 6) that hybridization slightly increased the density of the coconut fiber reinforced polymer composites. The density of the fiber reinforced polymer composites (FRPCs) decreased from $0.96 \mathrm{~g} / \mathrm{cm}^{3}$ at $0 \mathrm{Wt}$. \% of coconut fiber addition to $0.72 \mathrm{~g} / \mathrm{cm}^{3}$ at $30 \mathrm{Wt}$. \% for un-hybridized bumper samples, and $0.99 \mathrm{~g} / \mathrm{cm}^{3}$ at $0 \mathrm{Wt}$. $\%$ to $0.76 \mathrm{~g} / \mathrm{cm}^{3}$ at $30 \mathrm{Wt}$. \% for the hybridized bumper samples. This is following the study investigated earlier by Zhang 2006; Suddin et al, 2004; Ping et al, 2002. However, the densities obtained are within the recommended standard for the low speed impact bumper.

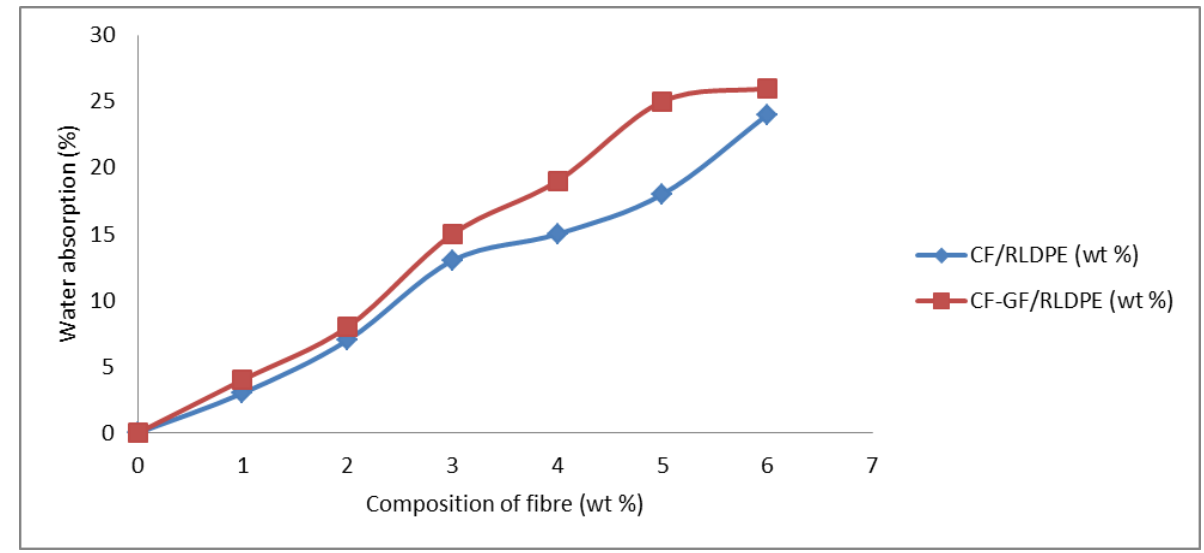

Fig 7. Influance of fibre composition on water absorption

The low level of water absorption (Figure 7) recorded may be due to the addition of glass fibers to the natural fibers, and the surface treatment of the natural fibers with alkali $(\mathrm{NaOH})$ solution. A similar observation was also reported [9] for other natural fibers. This increased interfacial bonding between the RLDPE and the fibers, thus leading to a decrease in the porosity level, hence the reported solubility values of the composites. The swelling that occurs during the water absorption may be due to the release of compression stresses imparted to the composites during the pressing of material in the hot press. The results obtained for CF-GF/RLDPE composites are within the recommended standard for a car bumper. Figure 8 shows the variation of tensile strength concerning the percentage composition of the fiber. 


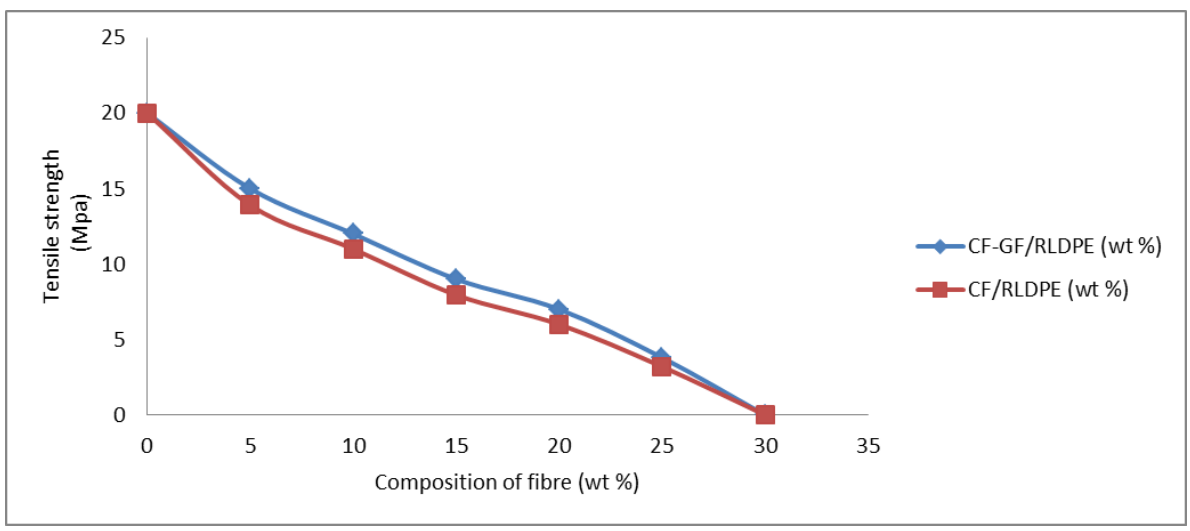

Fig 8. Influance of fibre composition on tensile strength

The tensile strength of the natural fiber reinforced polymer composites decreases as the composition of fiber in the matrix increases and vice-versa. The tensile strength decreased from $20.0 \mathrm{MPa}$ at $0 \mathrm{Wt}$. \% fiber addition to a minimum of $2.9 \mathrm{MPa}$ at $30 \mathrm{Wt}$. $\%$ for both the hybridized and the un-hybridized natural fiber reinforced composites. However, within this range, hybridization increases the tensile strength of the CF/RLDPE composites slightly. The tensile strength and moduli of the CF-GF/RLDPE composites showed higher values than CF/RLDPE composites because of the hybridization of the coconut fiber with glass fiber. This accounts for the excellent distribution and dispersion of the fibers in the RLDPE matrix resulting in strong fiberRLDPE interaction. Nevertheless, the tensile strength obtained in this study remained within acceptable levels for car bumper [8,12,15]. Figure 9 shows the variation of Modulus of rupture versus percentage composition of the fiber.

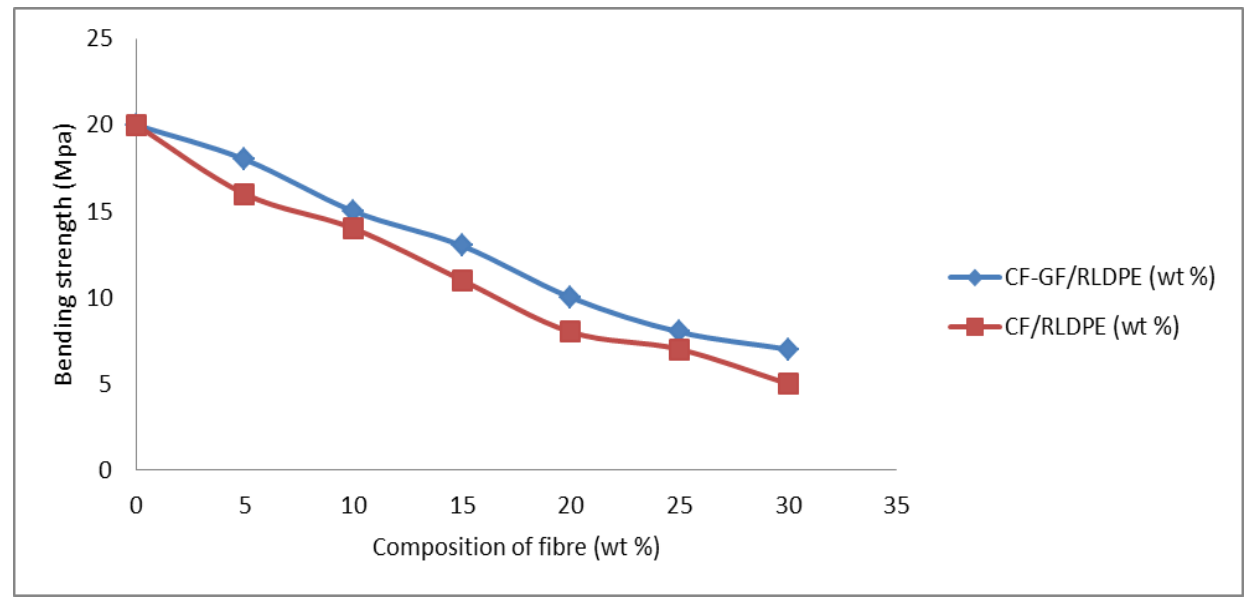

Fig 9. Influance of fibre composition on bending strength 
The bending strengths of the hybridized and the un-hybridized composites were obtained experimentally from the bend tests. The graph is shown in (Figure 9). It is interesting to note that bending strength increased with an increase in the reinforcement fiber content in the RLDPE matrix. For example, the bending strength of $13.0 \mathrm{MPa}$ was recorded for CF/RLDPE (un-hybridized) composite and 14.70 MPa for CF-GF/RLDPE (hybridized) composite at $10 \mathrm{Wt}$ \% fiber composition. There is an improvement in the bending strength of the composite as particle weight fraction increases. The random planer arrangement of the fibers is likely to lead to rigidity and better absorption of compressive forces, leading to an increase in overall bending strength. Figure 9 indicates the variation of Impact strength versus percentage composition of the fiber.

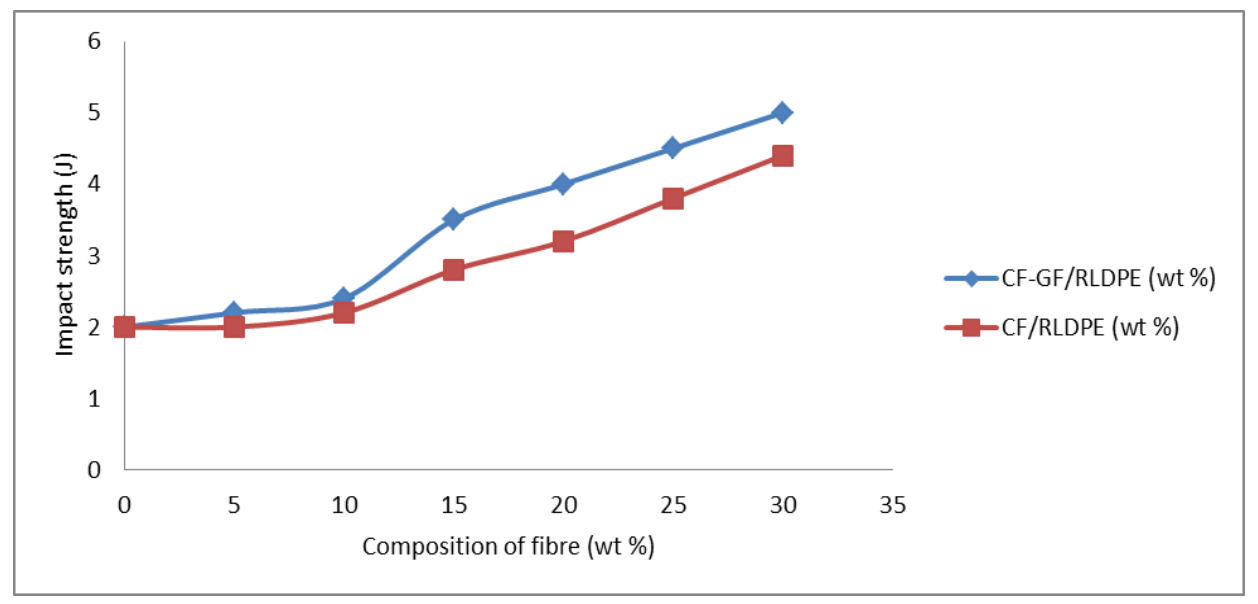

Fig 10. Influance of fibre composition on impact strength

The results of the impact strength show that the impact strength of the coconut fiber reinforced low density polyethylene composites slightly increased with an increase in glass fiber addition (Figure 10). High strain rates or impact loads may be expected in many engineering applications of polymer composite materials. The suitability of a polymer composite for such applications should, therefore, be determined not only by usual design parameters but by its impact or energy absorption. The steep increase in the impact strength composites could be attributed to hybridization and the presence of fibers well bonding by the RLDPE, which leads to an increase in impact strength. The steep improvement in the impact energy of the composites could be attributed to good interfacial bonding between the matrix and the fibers, which was achieved by surface treatment of the fibers with alkali $(\mathrm{NaOH})$ solution. These results are in agreement with the work of other researchers $[8,12,14]$. Figure 11 shows the variation in hardness value in relation to the percentage composition of the fiber. 


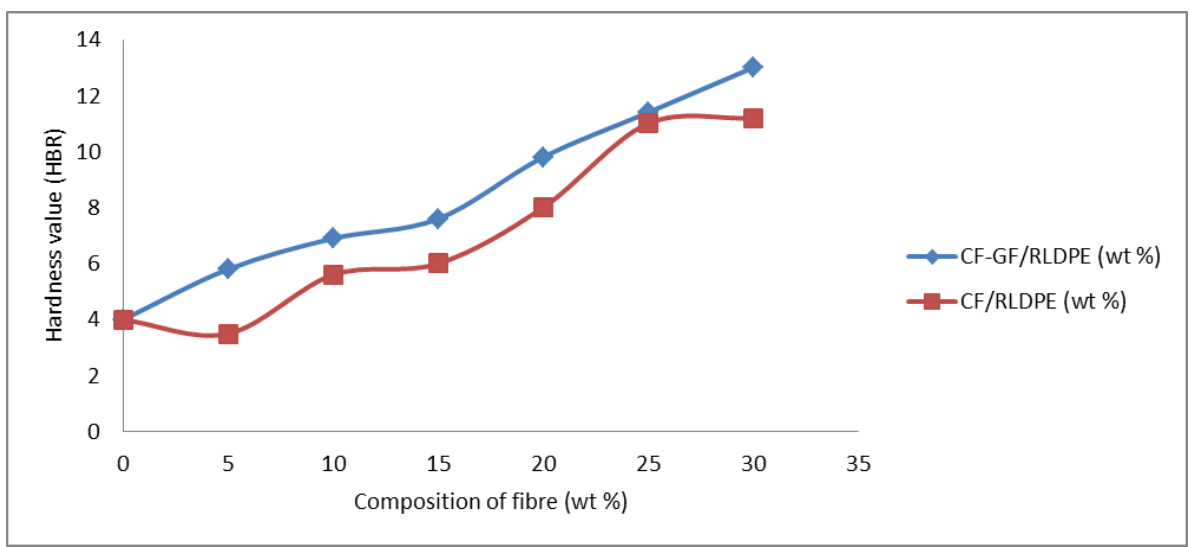

Fig 11. Influance of fibre composition on hardness values

The results obtained show that the hardness values of the composite samples increases as the percentage of fiber addition increases in the RLDPE matrix (figure 11). This is due to an increase in the percentage of the hard and brittle phase of the ceramics body in the polymer matrix. In comparison with the unreinforced RLDPE matrix, a substantial improvement in hardness values was obtained in the reinforced polymer matrix. This is in line with the earlier researches $[8,12]$. It can be deduced from the graph above that hybridization increases the hardness of the natural fiber-reinforced composites. However, the mechanical properties of the hybridized bumper samples fall within the recommended limits for standard car bumper $[14,15]$.

\section{Summary}

For this study, experimental techniques have been comprehensively employed to characterize the microstructures, physic-chemical properties of hybridized and unhybridized natural fiber-reinforced low density polyethylene composites using a varying composition of coconut and glass fibers. The following conclusions were drawn:

1) The work shows the successful development of composites of coconut fiber (CF) hybridized with glass fiber (GF) and reinforced low density polyethylene (RLDPE) binder by the simple compression molding technique.

2) The tensile strength of the developed composites decreased as the fractional weight of the reinforcement increased for both the hybridized and the unhybridized composites. This was in line with flexural strength behavior. Both properties increased with the fiber length of the reinforcement fibers

3) The impact strength of the composite materials increased with increasing weight fraction of reinforcement fibers. Hybridized samples showed higher strength than the un-hybridized composites. A similar observation was made for their hardness properties. 
4) Better interfacial bonding between reinforcement and matrix was observed with 15,25 , and $30 \%$ wt CF-GF hybrid composite leading to good mechanical properties for the hybridized composites.

5) Based on the results obtained in this study, these grades of composites can be used in the production of a low strength car bumper

\section{References}

[1] W. S. Arnold, I.H. Marshall, J. Wood: Composite Structures, 16 (1990) 85-101.

[2] S. S. Cheon, J. H. Choi, D. G Lee: Composite Structures, 32 (1995) 491-499.

[3] M. M. Davido, S. M. Sapuan, D. Ahmad, A. Ali, K. Abdan, M. Jonoobi: Journal, Materials and Design, 31 (10) (2010) 4927-4932.

[4] B. P. Minaudo, J. Rawson, M. Montone: SAE Technical paper 970483 (1997).

[5] C. L. Clark, C. K. Bals, M. A. Layson: SAE Technical Paper 910049 (1991).

[6] B. Gilliard, W. Bassett, E. Haque, T. Lewis, D. Featherman, C. Johnson: SAE Technical Paper, 1 (1999) 10-14.

[7] J. Rawson: SA Technical Paper 1999-01-1015 (1999)

[8] M. N. Suddin, M. S. Salit, N. Ismail, M. A. Maleque, S. Zainuddin: Suranaree J. Science Technology. 12, 1 (2004) 39-45.

[9] M. M. Davoodi: Elsevier Materials and Design, 29 (2008) 1447-1452.

[10] ASTM D578 / D578M - 18 Standard Specifications for Glass Fiber Strands. Book of Standards Volume: 07.01

[11] M. Sumaila, I. Amber, M. Bawa: Asian Journal of Natural and Applied Sciences, 2 (1) (2013) 39-49.

[12] BS EN 319:1993: Particleboards and fibreboards-Determination of Tensile Strength Perpendicular to the Plane of the Board. The British Standards Institution, London.

[13] Y. Zhang, Y: Elsevier Materials and Design, PR China, 27 (2006) 64-68.

[14] C. G. E. Ping, N. Wang, C. Stephen, Y. Lu: Journal of Computing and Information Science in Engineering, 2 (2002) 141.

[15] D. J. Andrea, W.R. Brown: W.R., (1993). Material Selection Processes in Automotive Industry, Michigan Transportation Research Institute, USA, 34.

[16] R. Hosseinzadeh, M. M. Shokrieh, L. B. Lessard: Composite Structures, 68 (2004) 419-427.

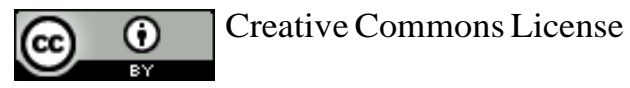

This work is licensed under a Creative Commons Attribution 4.0 International License. 